\title{
Application of the Jacobi-Davidson method to spectral calculations in magnetohydrodynamics
}

\author{
A.J.C. Beliën ${ }^{1}$, B. van der Holst ${ }^{1}$, M. Nool ${ }^{2}$, A. van der Ploeg ${ }^{3}$, and \\ J.P. Goedbloed ${ }^{1}$ \\ 1 FOM-Instituut voor Plasmafysica Rijnhuizen, Postbus 1207, \\ 3430 BE Nieuwegein, The Netherlands \\ 2 Centre for Mathematics and Computer Science, Postbus 94079, \\ 1090 GB Amsterdam, The Netherlands \\ 3 MARIN, Postbus 28, 6700 AA Wageningen, The Netherlands
}

Presenting author: A..J.C. Beliën

E-mail: belien@rijnh.nl

Tel.: +31 30-6096954

Fax : +31 20-6031204 


\title{
Application of the Jacobi-Davidson method to spectral calculations in magnetohydrodynamics
}

\author{
A.J.C. Beliën ${ }^{1}$, B. van der Holst ${ }^{1}$, M. Nool ${ }^{2}$, A. van der Ploeg ${ }^{3}$, and \\ J.P. Goedbloed ${ }^{1}$ \\ 1 FOM-Instituut voor Plasmafysica Rijnhuizen, Postbus 1207, \\ 3430 BE Nieuwegein, The Netherlands \\ 2 Centre for Mathematics and Computer Science, Postbus 94079, \\ 1090 GB Amsterdam, The Netherlands \\ 3 MARIN, Postbus 28, 6700 AA Wageningen, The Netherlands
}

\begin{abstract}
For the solution of the generalized complex non-Hermitian eigenvalue problems $A x=\lambda B x$ occurring in the spectral study of linearized resistive magnetohydrodynamics (MHD) a new parallel solver based on the recently developed Jacobi-Davidson [18] method has been developed. A brief presentation of the implementation of the solver is given here. The new solver is very well suited for the computation of some selected interior eigenvalues related to the resistive Alfvén wave spectrum and is well parallelizable. All features of the spectrum are easily and accurately computed with only a few target shifts.
\end{abstract}

\section{Introduction}

Plasma is the single most occurring state of matter in the universe. It is characterized by such high temperatures that almost all atoms are ionized completely. In most situations where plasmas occur a magnetic field is present which will interact with the charged plasma particles. On a global scale, i.e., on time and length scales much larger than typical kinetic time and length scales, the interaction of plasma with magnetic fields can be described by magnetohydrodynamics (MHD). This theory is scale invariant so that it is applicable to such diverse and utterly different objects as stellar winds, coronal loops, and thermonuclear fusion plasmas in tokamaks, to name a few.

A key aspect of the MHD analysis of plasmas is the study of waves and instabilities. In thermonuclear fusion plasmas the goal is to confine a dense hot plasma for as long as is necessary to reach ignition. MHD instabilities limit the densities that can be obtained and, hence, have a negative impact on fusion operation. In fact, the main activity of the MHD fusion theory in the last thirty years has been to increase this limit by optimizing the plasma profiles and plasma cross-section with respect to stability, see for example Ref. [5]. From a plasma physics point of view this emphasis on (in)stability does not do justice to the importance of the stable part of the spectrum. Besides the fact that a multitude of observed phenomena in astrophysical and laboratory plasmas are wave-like, detailed knowledge of the spectrum of waves allows for MHD spectroscopy (see, 
e.g., Refs. [10, 1, 9, 2, 4]). Free oscillations (waves) play a dominant role in MHD spectroscopy. It is the computation of these free eigen-oscillations that we are interested in in this paper.

Mathematically, free oscillations and instabilities of a resistive MHD plasma are described by a large complex non-Hermitian generalized eigenvalue problem. Until recently, the large scale spectral codes CASTOR [16, 12] and POLLUX [6] that we employ for the computation of tokamak and coronal loop spectra calculated them either by solving for the whole spectrum with a direct dense matrix method like QR or using inverse vector iteration for a selection of eigenpair solutions in the neighborhood of a target value [11]. The QR method is limited to very coarse meshes (due to its storage and computation requirements) and large values of the resistivity (to get reasonably converged results). Therefore, the eigenvalues obtained with QR are normally used as initial guesses for the inverse vector iteration only. For the application of interest, viz., the computation of the resistive Alfvén wave spectrum for realistic small values of the resistivity $\left(\eta<10^{-7}\right)$, the QR eigenvalues are very poor initial guesses. The original versions of the CASTOR and POLLUX codes contained a two-sided Lanczos solver with no orthogonalization $[3,11]$. Since no orthogonalization is used criteria are implemented that do away with occurring spurious eigenvalues. However, these criteria are not bullet-proof. Not all spurious eigenvalues are removed and occasionally proper eigenvalues are labeled as spurious and removed. Furthermore, the accuracy of the computed eigenvalues is generally poor and the inverse vector iteration has to be used to get good converged eigenvalues. We needed a more robust iterative solver to obtain several eigenvalues at once within a specified region of the complex plane. We have opted for the Jacobi-Davidson method.

The Jacobi-Davidson (JD) subspace iteration method is a new and powerful technique for solving non-symmetric eigenvalue problems in a sequence of steps $[18,19,20]$. It is extremely suitable for solving the resistive Alfvén spectrum since such a spectrum consists of many complex branches. To obtain converged results on the interior Alfvén modes, an effective search mechanism is highly desirable. Such a search mechanism is provided by the JD algorithm. Compared with the Arnoldi method, where the projection matrices are always upper Hessenberg, these matrices in JD are transformed to upper Hessenberg each step. This allows the restart technique of JD to be simpler. Another difference is the fact that by selecting the Ritz value that has the maximum absolute value the JD method can be accelerated (see also [14]).

In this paper we briefly describe the JD method and its parallel implementation for use in MHD spectral computations and apply it to the calculations of some resistive MHD spectra in tokamaks.

\section{Equilibrium, spectral equations and core numerical problem}

For the description of small amplitude waves and instabilities we exploit the linearized resistive MHD equations for the evolution of the density $\rho$, velocity $\mathbf{v}$, 
pressure $p$, and magnetic field $\mathbf{B}$, where resistivity is denoted by $\eta$ :

$$
\begin{aligned}
\frac{\partial \rho_{1}}{\partial t} & =-\nabla \cdot\left(\rho_{0} \mathbf{v}_{1}\right), \\
\rho_{0} \frac{\partial \mathbf{v}_{1}}{\partial t} & =-\nabla p_{1}+\left(\nabla \times \mathbf{B}_{0}\right) \times \mathbf{B}_{1}+\left(\nabla \times \mathbf{B}_{1}\right) \times \mathbf{B}_{0}, \\
\frac{\partial p_{1}}{\partial t} & =-\mathbf{v}_{1} \cdot \nabla p_{0}-\gamma p_{0} \nabla \cdot \mathbf{v}_{1}+(\gamma-1) \eta\left(\nabla \times \mathbf{B}_{1}\right)^{2}, \\
\frac{\partial \mathbf{B}_{1}}{\partial t} & =\nabla \times\left(\mathbf{v}_{1} \times \mathbf{B}_{0}\right)-\nabla \times\left(\eta \nabla \times B_{1}\right) .
\end{aligned}
$$

The subscripts 0 and 1 indicate equilibrium and perturbation quantities, respectively. Together with boundary conditions this constitutes the spectral MHD formulation. The equilibrium quantities obey the ideal static force balance:

$$
\nabla p_{0}=\left(\nabla \times \mathbf{B}_{0}\right) \times \mathbf{B}_{0}, \quad \nabla \cdot \mathbf{B}_{0}=0 .
$$

Discretization of Eqs.(1-4) using finite elements in the direction across the magnetic field and Fourier harmonics in the poloidal and toroidal directions results in a generalized eigenvalue problem for the case of free plasma oscillations and instabilities

$$
\mathbf{A x}=\lambda \mathbf{B} \mathbf{x}
$$

where $\lambda$ is an eigenvalue. Its imaginary part describes oscillations, its real part instabilities and damping. Matrix $\mathbf{B}$ is a complex Hermitian positive definite block tridiagonal matrix while matrix $\mathbf{A}$ is a complex non-Hermitian block tridiagonal matrix. Both $\mathbf{A}$ and $\mathbf{B}$ are of size $M \times M$ with $M=n N$. The block size $n$ is a always a multiple of 16 . The number of diagonal blocks is denoted by $N$.

\section{An eigenvalue solver based on the Jacobi-Davidson algorithm}

The Jacobi-Davidson method $[18,19,20]$ is based on two concepts: the application of a Ritz-Galerkin approach to the eigenvalue problem (6) with respect to a subspace spanned by an orthonormal basis of low dimensionality, and the construction of a new search vector orthogonal to the eigenvector approximations that have been obtained so far.

To obtain a new search direction the JD algorithm solves a system of linear equations called the correction equation. An appropriate preconditioner has to be applied to obtain fast convergence such that the correction equation can be solved to some modest accuracy using only a few steps of, e.g., GMRES [17]. Because $\mathbf{A}-\sigma \mathbf{B}$, where $\sigma$ is the target value in the vicinity of which eigenvalues are sought, has relatively full blocks, due to the structure of the MHD equations, a parallel complete block LU-decomposition has been shown to be a fast and robust method to solve the correction equation [14].

Both the construction and the application of $\mathrm{L}$ and $\mathrm{U}$ can be parallelized efficiently by, prior to decomposition, reordering block rows and columns based on 
Domain Decomposition and block Cyclic Reduction (DDCR). Due to the special block tridiagonal form of $\mathbf{A}-\sigma \mathbf{B}$ its LU-decomposition can be constructed without excessive memory requirements or a large number of operations. On account of the cyclic reduction part of the decomposition, which starts on all processors, while half of the active processors becomes idle after each step, we may not expect linear speed-up. However, the overall performance of DDCR is quite good due to the domain decomposition part (see [15]). Since the construction and application of the LU-decomposition form the most time consuming part this guarantees good overall parallel performance as well. In Fig. 1, we show the Gflop/s rate for the DDCR-decomposition and the corresponding solution process SOLDDCR on a CRAY-T3E for different block sizes and number of diagonal blocks. From this figure it is clear that the total number of diagonal blocks, $N$, has a far greater influence on the scalability than the block size $n$.
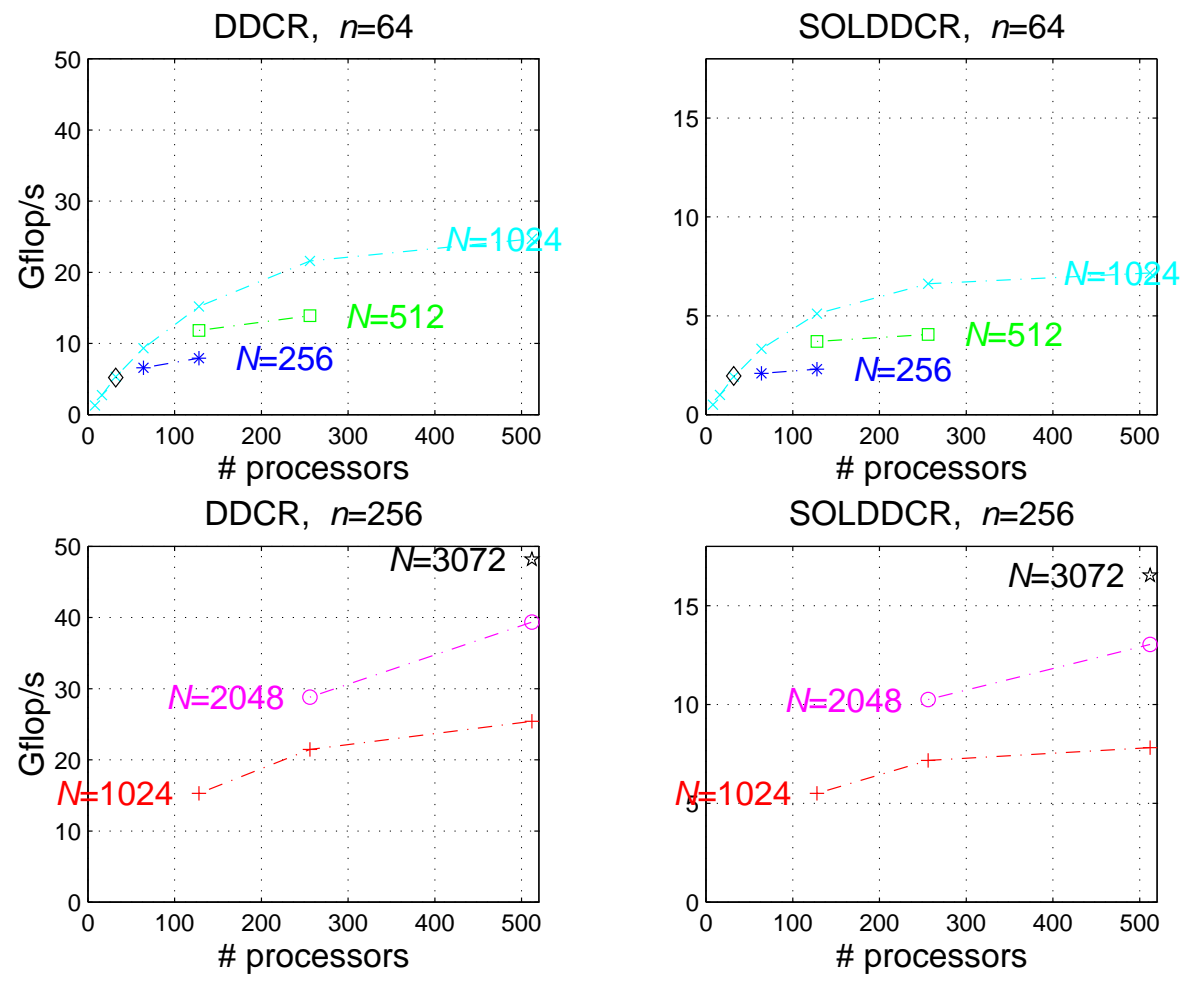

Fig. 1. Gflop/s-rate on a CRAY-T3E, with a single-node peak performance of 600 $\mathrm{Mflop} / \mathrm{s}$, for the construction (left) and application (right) of the LU-decomposition based on a domain decomposition and block cyclic reduction method (DDCR). The results are obtained for block sizes $n=64$ and $n=256$. The number of diagonal blocks varies from $N=256$ till $N=3072$. 
With the definition of $Q \equiv(L U)^{-1} B$ we apply JD to the standard eigenvalue problem

$$
\mathbf{Q x}=\mu \mathbf{x}
$$

with the inverse shifted eigenvalues $\mu=1 /(\lambda-\sigma)$ which automatically make the desired eigenvalues $\lambda$, close to the target $\sigma$, the dominant ones and, hence, 'easy' to compute. When the target $\sigma$ is close to an eigenvalue, small pivot elements can be generated in the LU-decomposition of $\mathbf{A}-\sigma \mathbf{B}$ and the application of $Q$ may influence the computed spectrum. In such cases using smaller tolerances or exploiting different target values can give information on the accuracy. It should be noted that numerical experiments with a similar algorithm using harmonic Ritz values applied to the generalized eigenvalue problem is a more promising approach [13,18]. The main advantage of that approach is that the LU-decomposition is only used as a preconditioner and not as a shift and invert operator. However, the method exploiting harmonic Ritz values consumes more memory and costs approximately $20 \%$ more execution time per Jacobi-Davidson iteration step.

At the $k$-th step of the JD iteration algorithm the approximation of the eigenvector can be written as $\mathbf{V}_{k} \cdot \mathbf{s}$ where $\mathbf{V}_{k}$ is the $M \times k$ matrix whose columns are the $k$ search vectors. The search directions have been made orthonormal to each other using the Modified Gram-Schmidt procedure. The vector $\mathbf{s}$ and the approximation $\theta$ of an eigenvalue of $\mathbf{V}_{k} \cdot \mathbf{s}$ are constructed such that the residual $r=\left(\mathbf{Q} \cdot \mathbf{V}_{k}-\theta \mathbf{V}_{k}\right) \cdot \mathbf{s}$ is orthogonal to the $k$ search directions. The Rayleigh-Ritz requirement then leads to

$$
\mathbf{V}_{k}^{*} \cdot \mathbf{Q} \cdot \mathbf{V}_{k} \cdot \mathbf{s}=\theta \mathbf{s},
$$

where $\theta$ and $\mathbf{s}$ are an eigenpair of the small matrix $\mathbf{V}_{k}^{*} \cdot \mathbf{Q} \cdot \mathbf{V}_{k}$ of size $k$. A proper restart technique keeps the size of the matrix on the left-hand side very small compared to $M$ enabling the solution with a direct method, for example QR.

The calculation of the eigenvalues and eigenvectors of the small projected system has not been parallelized. Actually, it is performed by all processors, because then after the calculation on each processor all information is available without communication. The projected system (8) of the Jacobi-Davidson process contains a lot of information about the eigenvalues in the neighborhood of the target $\sigma$. The question arises how much information may be thrown away when a restart is performed, necessarily to keep the projected system small, without slowing down the convergence behavior too much. Obviously, in the parallel case the size of the projected system plays even a more important role in wall clock time than in the sequential case. In [13], it is shown that it depends on the value $M / p$, where $p$ is the number of processors, whether restarts lead to a reduction in the wall clock time for the parallel JD process.

The maximum problem size depends on the number of processors $p$ and the total amount of memory per processor. Our parallel implementation requires per processor

$$
128 N_{p} n^{2}+16 N_{p}\left(3 m+N_{\mathrm{ev}}+7\right)+96 n^{2}+64 m(m+1) \text { bytes, }
$$


as is shown in [13], where $N_{p}$ denotes the maximum number of diagonal blocks on one processor, $m$ the maximum allowed size of the projected system and $N_{\mathrm{ev}}$ stands for the desired number of eigenvalues.

\section{Application to resistive toroidal spectra}

As an example, we have used the JD solver to solve the stable resistive spectrum of a tokamak with circular cross-section and an inverse aspect ratio $\epsilon=0.2$. The results are shown in Fig. 2.

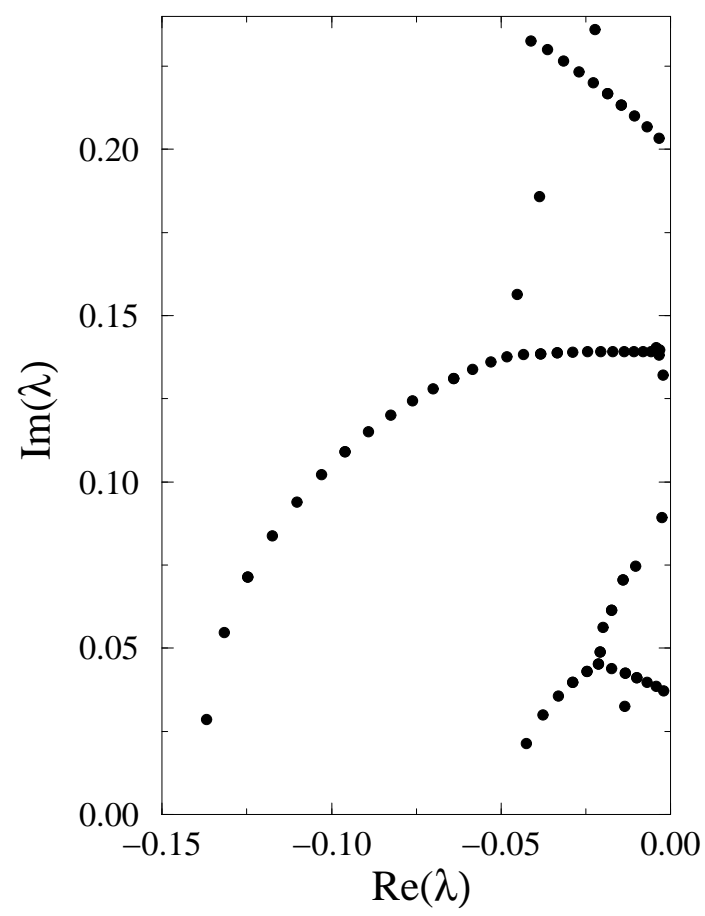

Fig. 2. The resistive spectrum for unit toroidal mode number, inverse aspect ratio $\epsilon=0.2$, and resistivity $\eta=2.5 \times 10^{-5}$. Purely damped eigenmodes have been left out.

The resistive spectrum was calculated with 1000 radial points and 4 poloidal harmonics, corresponding to matrices $\mathbf{A}$ and $\mathbf{B}$ with a block size of $n=64$ and $N=1000$ diagonal blocks. The acceptance criterium of a Ritz eigenpair leading to a eigenpair of the original problem (6) is that the 2-norm of the residual is smaller than $10^{-6}$ times the eigenvalue. The implementation of the JD solver is such that it terminates when $N_{\mathrm{ev}}$ are found or the maximum number of iterations is surpassed. The computations were done with $N_{\mathrm{ev}}=10$ to keep the size of 
the matrix occurring in Eq. (8) as small as possible. Several target shifts are necessary to map out the spectrum and many eigenvalues were found by more than one shift. If the target is not well-chosen, it may happen that less than $N_{\mathrm{ev}}$ eigenvalues will be found. In that case a new target based on the obtained spectrum must be taken rather than to increase the number of iterations.

Inspection of the number of radial nodes of the eigenfunctions can be used to ensure that all eigenvalues have been found. For smaller resistivity the topology of the spectrum remains the same but the number of eigenvalues situated on the curves shown in Fig. 2 increases (the number is proportional to $\eta^{-1 / 2}$ ). One then has the choice of enlarging $N_{\mathrm{ev}}$ so that more eigenvalues can be found for one specified target shift at the expense of larger matrices in the Rayleigh-Ritz part, or using more target shifts. The fact that new target shifts require new LU-decompositions, which are the computationally most intensive operations, (though well parallelizable) the first option seems the best.

Physically, the eigenvalues are converged as well: taking more poloidal harmonics or more radial grid points into account results in changes that are smaller than the acceptance criterium for the Ritz pairs. The physical interpretation of resistive spectra, like the one shown in Fig. 2, can be found in Ref. [7].

\section{Conclusions and Outlook}

The Jacobi-Davidson method appears to be an excellent method for parallel computation of resistive Alfvén spectra that occur in studies of the linearized interaction between plasmas and magnetic fields. The method discussed in this paper is based on solving projected eigenvalue problems of an order typically less than 30 .

Within Jacobi-Davidson a parallel method to compute the action of the inverse of the block-tridiagonal matrix $A-\sigma B$ is used. In this approach, called DDCR, a block-reordering based on a combination of Domain Decomposition and Cyclic Reduction is combined with a complete block-tridiagonal LU decomposition of $A-\sigma B$, so $L U=A-\sigma B$. Both the construction of $L$ and $U$ and the triangular solves parallelize well.

We have successfully applied the JD-solver to the problem of computing branches of the stable resistive Alfvén spectrum with only a few target values $\sigma$ (smaller resistivity requires more targets) and with an accuracy of $10^{-6}$.

This has given us enough confidence to endeavor into the much more complex field of computing the spectra for plasmas with background flows, which is presently under taken [8].

Acknowledgments. This work was performed as part of the research programme of the 'Stichting voor Fundamenteel Onderzoek der Materie' (FOM) with financial support from the 'Nederlandse Organisatie voor Wetenschappelijk Onderzoek' (NWO). Part of this work is done in the project on 'Parallel Computational Magneto-Fluid Dynamics', funded by the NWO Priority Program on 
Massively Parallel Computing. Another part is sponsored by the 'Stichting Nationale Computerfaciliteiten' (NCF), also for the use of supercomputer facilities.

\section{References}

1. Beliën, A.J.C., Poedts, S., and Goedbloed, J.P., Phys. Rev. Lett. 76, 567 (1996).

2. Beliën, A.J.C., Poedts, S., and Goedbloed, J.P., Astron. Astrophys. 322, 995 (1997).

3. Cullum, J., Kerner, W., and Willoughby, R.A., IBM RC14190, February 1988, IBM T.J. Watson Research Center, Yorktown Heights, in Computer Physics Communications, 53 (North-Holland, Amsterdam, 1988).

4. De Ploey, A., van der Linden, R.A.M, and Beliën, A.J.C., to appear in Physics of Plasmas (2000).

5. Freidberg, J.P., "Ideal magnetohydrodynamics", Plenum Press, New York, 1987.

6. Halberstadt, G., and Goedbloed, J.P., Astron. Astrophys. 301, 559 \& 577 (1995).

7. van der Holst, B., Beliën, A.J.C., Goedbloed, J.P., Nool, M., and van der Ploeg, A., Physics of Plasmas 6, 1554 (1999).

8. van der Holst, B., Beliën, A.J.C., Goedbloed, J.P., "New Alfvén Continuum Gaps and Global Modes Induced by Toroidal Flow", Phys. Rev. Lett., to appear (March 2000).

9. Holties, H.A., Fasoli, A., Goedbloed, J.P., Huijsmans, G.T.A., and Kerner, W., Physics of Plasmas 4, 709 (1997).

10. Huijsmans, G.T.A., Kerner, W., Borba, D., Holties, H.A., and Goedbloed, J.P., Physics of Plasmas 2, 1605 (1995).

11. Kerner, W., J. Comput. Phys. 85, 1 (1989).

12. Kerner, W., Goedbloed, J.P., Huijsmans, G.T.A., Poedts, S., and Schwartz, E., J. Comput. Phys. 142, 271 (1998).

13. Nool, M., and van der Ploeg, A., "Parallel Jacobi-Davidson for Solving Generalized Eigenvalue Problems", in Palma, J.M.L.M., Dongarra, J., and Hernandez, V., editors, Proceedings of the Third International Meeting on Vector and Parallel Processing, volume 1573 of Lecture Notes in Computer Science, p. 58-71, SpringerVerlag, Berlin, 1999.

14. Nool, M., and van der Ploeg, A., "A Parallel Jacobi-Davidson-type Method for Solving Generalized Eigenvalue Problems in Magnetohydrodynamics" to appear in SIAM J. on Scientific Computing.

15. van der Ploeg, A., "Reordering Strategies and LU-decomposition of Block Tridiagonal Matrices for Parallel Processing", Technical Report NM-R9618, CWI, Amsterdam, October 1996.

16. Poedts, S., Kerner, W., Goedbloed, J.P., Keegan, B., Huijsmans, G.T.A., and Schwartz, E., Plasma Phys. Controlled Fusion 34, 1397 (1992).

17. Saad, Y., Schultz, M.H., SIAM Journal of Scientific and Statistical Computing, 17, 856, 1986.

18. Sleijpen, G.L.G., and van der Vorst, H.A., SIAM J. Matrix Anal. Appl. 17, 401 (1996).

19. Sleijpen, G.L.G., Booten, G.L., Fokkema, D.R., and van der Vorst, H.A., BIT, 36, 595 (1996).

20. Sleijpen, G.L.G., van der Vorst, H.A., and Bai, Z, Chapters 4, 5, 7, and 8 in "Templates for the Solution of Algebraic Eigenvalue Problems: A Practical Guide", eds. 
Bai, Z., Demmel, J., Dongarra, J., Ruhe, A., and van der Vorst, H.A, SIAM, to appear in 2000 .

This article was processed using the $\mathrm{LT}_{\mathrm{E}} \mathrm{X}$ macro package with LLNCS style 